\title{
SELFCONSISTENT CALCULATION OF ELECTRONIC SUPERSHELLS IN METAL CLUSTERS
}

\author{
O. Genzken and M. Brack
}

Inst. f. Theor. Physik, Universität Regensburg, D-8400 Regensburg, Germany

\begin{abstract}
We report on selfconsistent microscopic calculations of the electronic shell and supershell structure of sodium clusters with up to $N \sim 3000$ atoms. The spherical jellium model in local density approximation is used and the Kohn-Sham equations are solved numerically. The finite temperature of the valence electrons is included by treating them as a canonical subsystem embedded in the heat bath of the ions. In particular, we evaluate the total free energy $F(N)$ and investigate its fluctuating part, the shell-correction energy $\delta F(N)$, as a function of temperature $T$ and particle number $N$. We also discuss the second difference $\Delta_{2} F(N)=F(N+1)+$ $F(N-1)-2 F(N)$ and its relation to the recently measured cluster mass abundance spectra.
\end{abstract}

Metal clusters provide a unique example for the study of shell effects in finite fermion systems containing up to several thousand particles [1]. A selfconsistent microscopic description of such large systems is possible only in the so-called selfconsistent jellium model [2], where the interacting valence electrons move in the field of a uniformly charged sphere ('jellium') representing the ions. We report here on recent Kohn-Sham calculations for sodium clusters with up to $N \sim 3000$ atoms at finite temperatures [3].

Nishioka et al. [4], using a phenomenological Woods-Saxon potential fitted to the microscopic potentials of Ekardt [2], have drawn attention to the 'supershell structure' in the level density and the oscillating part of the total binding energy of clusters with $N$ up to 4000: a pronounced beating pattern in which the shell structure is enveloped by a slowly oscillating amplitude. This is, in fact, a very general feature of discrete eigenmodes in a cavity or in any steep potential confining many particles to a limited domain of space. Balian and Bloch have shown in their fundamental work [5] that the beating pattern of the level density in an infinite square well is explained by the superposition of amplitudes associated to closed classical trajectories; they 
reproduced the approximate shape of the exact level density by summing up only the contributions from triangular and squared classical orbits. Extending this method to smooth potentials, Strutinsky et al. [6] were able to explain the gross shell structure of atomic nuclei using realistic deformed shell model potentials. Nuclei, however, are not big enough to exhibit the 'supershell' beating of their shell structure.

The experimental observation of supershell structure in cluster expansion sources is inhibited by the fact that the clusters so produced have, at least initially, a finite temperature which tends to reduce the shell effects $[7,8,9]$. Nevertheless, in the newest sodium vapour expansion experiments of the Copenhagen - Orsay - Stuttgart collaboration [1], a supershell beating in the mass abundance spectrum of sodium clusters has been put into evidence. We shall show here that the experimental results can be explained semi-quantitatively in the selfconsistent jellium model, if the effect of finite temperature on the valence electrons is properly included.

We use the finite-temperature density functional theory in the Kohn-Sham (KS) approach [10], employing the spherical jellium model and the local-density functional for exchange and correlations by Gunnarsson and Lundqvist [11]. The Wigner-Seitz radius of bulk sodium, $r_{s}=3.96$ a.u., is used; otherwise our calculations are completely parameter free. We treat the valence electrons as a canonical ensemble in the heat bath of the ions and minimize the Helmholtz free energy $F(N)=E(N)-T S(N)$ of a cluster with $N$ atoms, where $E$ is its total internal energy and $S$ the entropy of the electrons at a given temperature $T$. (See Ref. [9] for details and, in particular, for a fast algorithm for the exact calculation of the canonical partition function.) A canonical treatment with exactly conserved particle number $N$ is important here since we investigate quantities like $\Delta_{2} F(N)=F(N+1)+F(N-1)-2 F(N)$ which are very sensitive to temperature effects through the entropy part $-T S$ : the large degeneracies of the spherical magic shells lead to large entropies even at small temperatures.

The quantity $\Delta_{2} E(N)=\Delta_{2} F(N)(T=0)$ has often been taken as a measure for the stability of the cluster: since it represents the curvature of the total binding energy as a function of $N$, it is particularly large for the 'magic' systems which have a strongly negative shell correction. It has furthermore been argued [7] that if the evaporation process, which takes place immediately after the adiabatic expansion, is responsible for enhancing the most stable clusters in the final mass yield, $\Delta_{2} F(N)$ 
should be proportional to $-\Delta_{1} \ln I_{N}$, where $I_{N}$ is the fluctuating part of the observed mass yield. In Refs. $[8,9]$ it has been shown that $\Delta_{2} F(N)$ decreases very fast for $N \sim 100-400$ and becomes practically zero for $N \geq 500$ already at $T \gtrsim 400 \mathrm{~K}$. Therefore, the smoothing effect of a finite temperature on quantities like $\Delta_{2} F(N)$ is very crucial for the observability of shell structure and, in particular, the supershells in large metal clusters.

The temperature dependence of shell structure has been well studied in nuclei, both schematically [12] and in selfconsistent Hartree-Fock calculations [13]. In a schematic harmonic oscillator approximation, the amplitude of the shell-correction $\delta F-$ and thus also of the quantity $\Delta_{2} F(N)$ in which the average energies practically cancel - is found to go like $\delta F(T)=\delta F(0) \tau /(\operatorname{Sinh} \tau)$ with $\tau=2 \pi^{2} T / \hbar \omega$. Expanding for large temperatures and using $\hbar \omega \propto N^{-1 / 3}$, this gives a temperature suppression factor $\propto \exp \left(-N^{1 / 3}\right)$. Pedersen et al. [1] therefore multiplied the logarithmic derivatives of the mass yields $I_{N}$ by $\sqrt{N} \exp \left(c N^{1 / 3}\right)$, where $c$ is a constant containing an effective temperature, and the root factor compensates the decrease of the shell-correction at $T=0$ with increasing $N$ [12]. In the resulting plot, magic shell closures with $N$ up to $\sim 2720$ and a beating of the shell oscillations can clearly be seen (see also Fig. 2 below).

As an example of our theoretical results, we show in Fig. 1 the free energy shellcorrection $\delta F(N)$ versus $N^{1 / 3}$ at the three temperatures $T=0 \mathrm{~K}, 400 \mathrm{~K}$ and 600 K. [Hereby we simply used $\delta F(N)=F(N)-\bar{F}(N)$ with a liquid drop model type expansion for the average free energy, $\bar{F}(N)=e_{b} N+a_{s} N^{2 / 3}+a_{c} N^{1 / 3}$, determining $a_{s}$ and $a_{c}$ at each temperature by a simple eye fit such that $\delta F(N)$ is oscillating around zero. The bulk energy is fixed at its theoretical value $e_{b}=-2.2567 \mathrm{eV}$.]

The salient feature of the curves in Fig. 1 is the supershell beating of the otherwise quite regular shell structure. The $T=0$ curve is very similar to that obtained by Nishioka et al. [4] for a phenomenological Woods-Saxon potential; note that the present results are fully selfconsistent. The amplitude of the shell effects is clearly reduced with increasing temperature.

In Figure 2, we have reproduced the relevant figure from the experimental analysis of Pedersen et al. [1] and compare it to our theoretical results. Here the negative second difference $-\Delta_{2} F(N)$ is shown, multiplied by the same enhancement factor (with the value of $c$ readjusted by $\sim 10 \%$ ). In spite of the simplyfing assumptions 
underlying the identification of $-\Delta_{2} F(N)$ with $\Delta_{1} \ln I_{N}$ [7,9], the agreement of the two curves is striking. This demonstrates that the finite temperature of the valence electrons which alone contribute to the quantities shown here - the ionic parts of the free energies practically cancel in the differences $\Delta_{2} F(N)$ and $\Delta_{1} \ln I_{N}$ plays an essential role in the mass yields and can be correctly taken into account in selfconsistent KS calculations even in the simple jellium model.

Much remains, however, to be understood - in particular the value of the factor $c$ which, using the above harmonic oscillator estimates, is too large for the estimated temperatures $[1,7]$ of $\sim 400-500 \mathrm{~K}$. A more realistic study of the evaporation mechanism and, more generally, a non-equilibrium treatment of the ions' dynamics would be desirable to this aim.

We are grateful to S. Bjørnholm for many enlightening discussions and a continuing encouragement, and to $\mathrm{K}$. Hansen for important contributions at the early stages of our investigations.

\section{References}

[1] J. Pedersen, S. Bjørnholm, J. Borggreen, K. Hansen, T. P. Martin and H. D. Rasmussen, Nature 353, 733 (1991); T. P. Martin, S. Bjørnholm, J. Borggreen, C. Bréchignac, Ph. Cahuzac, K. Hansen and J. Pedersen, Chem. Phys. Lett. 186, 53 (1991); see also the talks of S. Bjørnholm and T. P. Martin, these Proceedings.

[2] W. Ekardt, Phys. Rev. B 29, 1558 (1984).

[3] O. Genzken and M. Brack, Phys. Rev. Lett. 76, 3286 (1991).

[4] H. Nishioka, K. Hansen, and B. R. Mottelson, Phys. Rev. B 42, 9377 (1990).

[5] R. Balian, C. Bloch, Ann. Phys. (N.Y.) 69, 76 (1971).

[6] V. M. Strutinsky, A. G. Magner, S. R. Ofengenden, and T. Døssing, Z. Phys. A 283, 269 (1977).

[7] S. Bjørnholm, J. Borggreen, O. Echt, K. Hansen, J. Pedersen, H. D. Rasmussen, Z. Phys. D 19, 47 (1991). 
[8] M. Brack, O. Genzken, and K. Hansen, Z. Phys. D 19, 51 (1991).

[9] M. Brack, O. Genzken, and K. Hansen, Z. Phys. D 21, 65 (1991).

[10] For a review on finite-temperature density functional theory, see U. Gupta and A. K. Rajagopal, Physics Reports 87, 259 (1982); for its justification for canonical systems, see R. Evans, Adv. in Phys. 28, 143 (1979).

[11] O. Gunnarsson and B. I. Lundqvist, Phys. Rev. B 13, 4274 (1976).

[12] A. Bohr, and B. M. Mottelson, Nuclear Structure II (Benjamin, 1975).

[13] See, e.g., M. Brack and P. Quentin, Nucl. Phys. A 361, 35 (1981), and earlier references quoted there. 

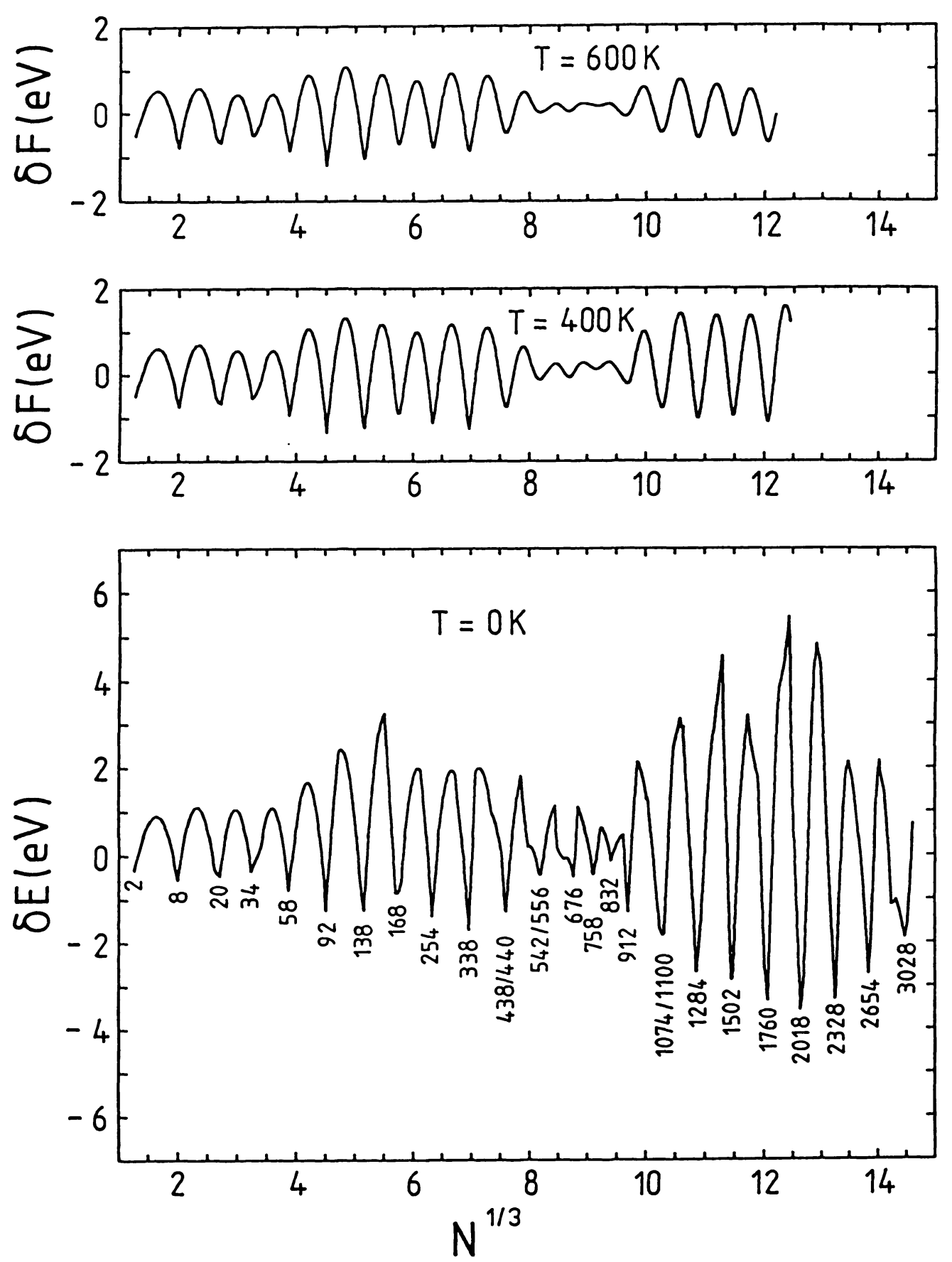

Figure 1: Free energy shell-correction $\delta F(N)=F(N)-\bar{F}(N)$ for spherical Na clusters versus $N^{1 / 3}$ for three different temperatures $T$, obtained in selfconsistent KS calculations [3]. LDM parameters used at $T=0 \mathrm{~K}: a_{s}=0.6259, a_{c}=0.2041$; at $T=400 \mathrm{~K}: a_{s}=0.5918, a_{c}=0.3796 ;$ and at $T=600 \mathrm{~K}: a_{s}=0.5755, a_{c}=0.4204$ (all in $\mathrm{eV}$ ). Numbers near the bottom are the magic numbers of filled major spherical electronic shells. 

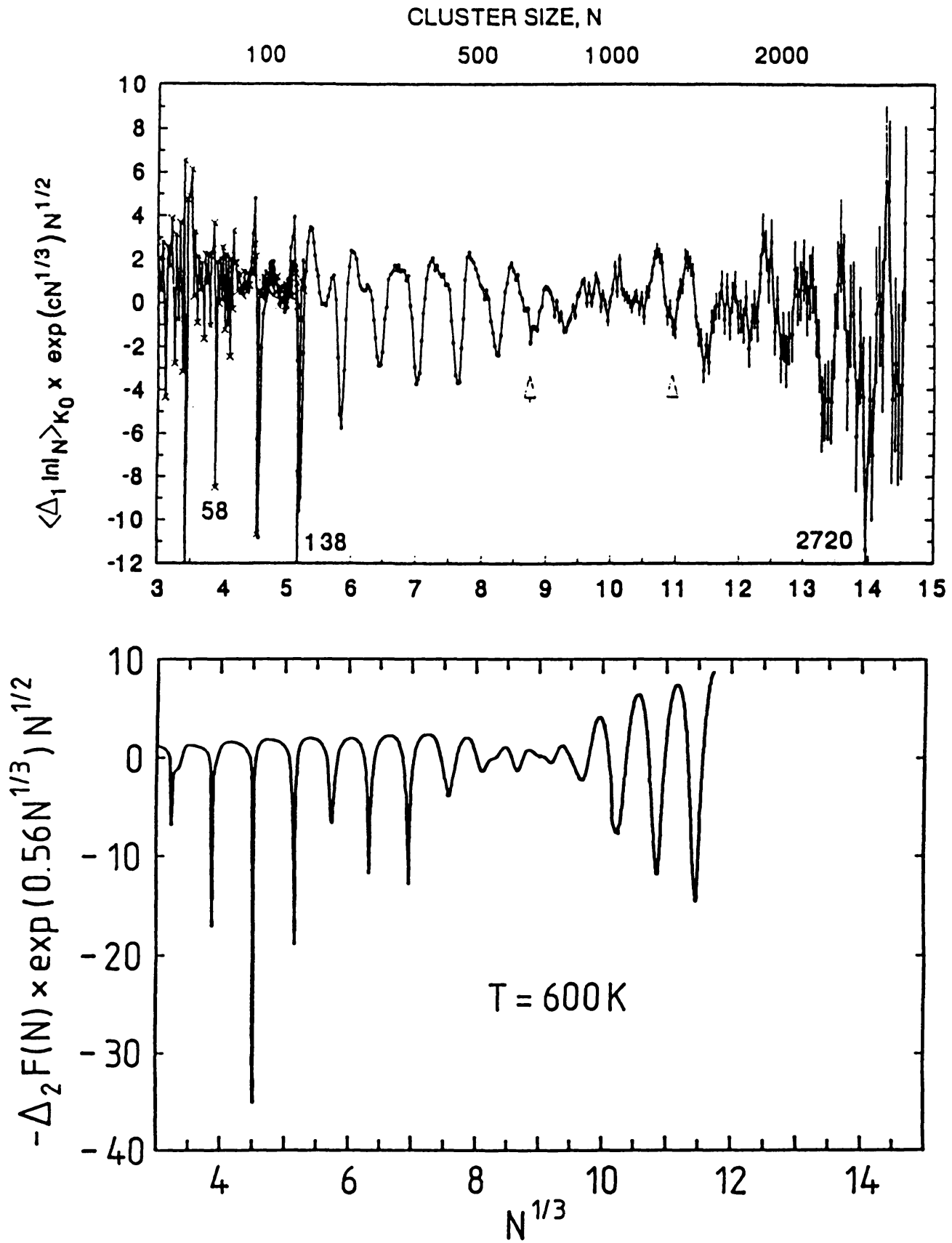

Figure 2: Uppcr part: Relative variation $\left\langle\Delta_{1} \ln I_{N}\right\rangle_{K_{0}}$ in expcrimental Na cluster abundance $I_{N}$ versus $N^{1 / 3}$ (Pedersen et al. [1]; see this reference for the details). Lower part: Negative second difference $-\Delta_{2} F(N)$ of free energy obtained in selfconsistent $\mathrm{KS}$ calculations [3] at $T=600 \mathrm{~K}$. Both quantities are enhanced by a $N$-dependent factor to compensate for the temperature suppression (see text). 